\title{
Research round-up
}

\section{Highlights from COPD trials. By Simon Makin}

\section{Genetic risk factors uncovered}

Two papers from large international collaborations have revealed genetic contributors to lung function and chronic obstructive pulmonary disease (COPD) that illuminate biological pathways and potential drug targets. They are some of the largest genetic studies of lung function and COPD so far.

The first, led by Nick Shrine at the University of Leicester, UK, assessed genetic markers linked with lung function in a genomewide association study of about 400,000 individuals of European ancestry. The researchers identified 279 genetic regions associated with lung function, 139 of which were new. The team showed that a combination of these variants could be used to predict COPD in both smokers and non-smokers. It also found that the risk for smokers could vary by as much as fivefold.

The findings highlight the importance of several biological pathways in COPD, including the development of cilia. These tiny hair-like structures help to clear mucus from the airways, and their function is known to be impaired in COPD. The research suggests that cilia dysfunction is not merely a consequence of COPD, but a driver of the disease. The team also examined associations between specific genetic variants and a wide range of disease traits, and found evidence that COPD shares some genetic determinants with autoimmune diseases.

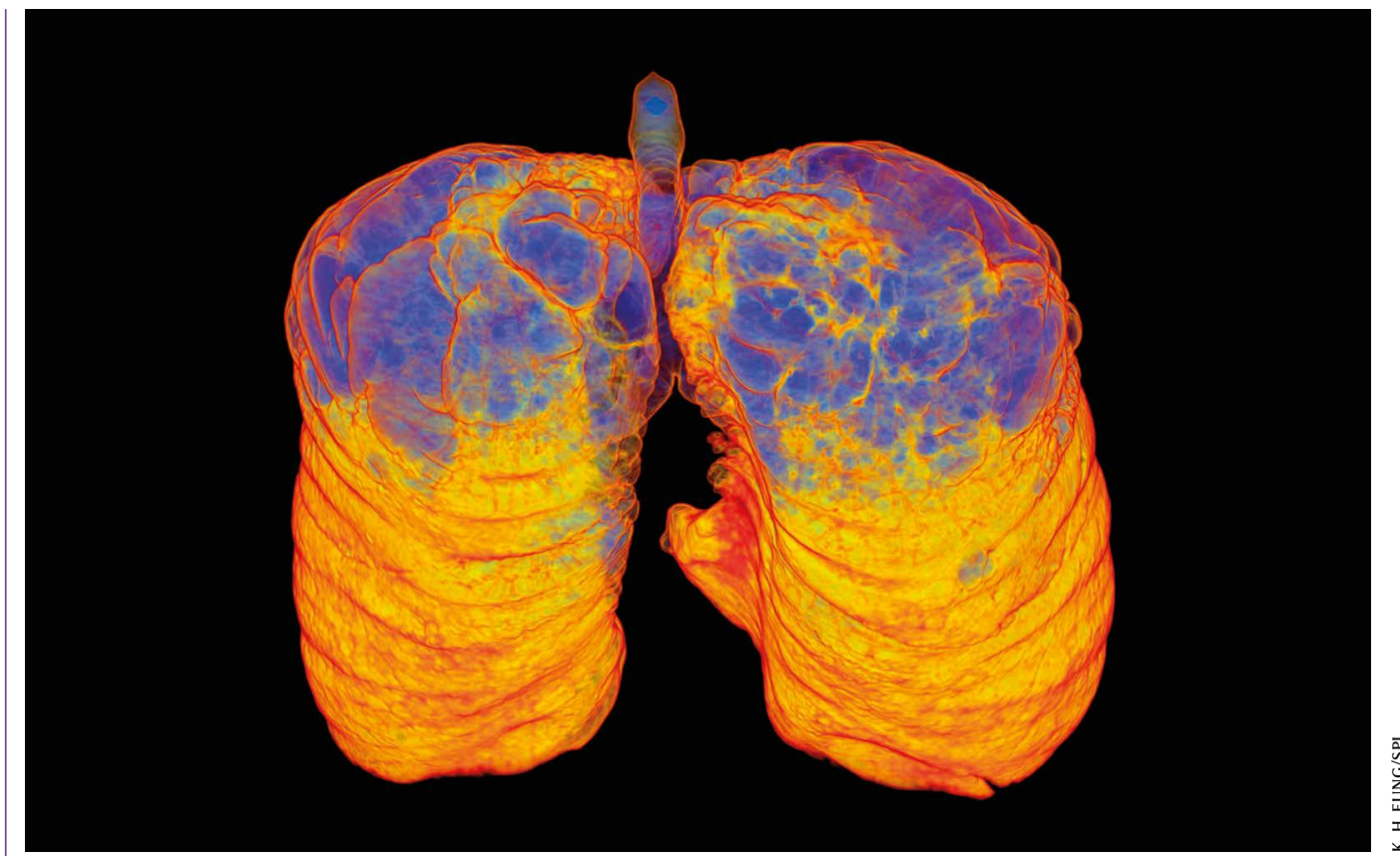

Computed tomography image of lungs showing signs of emphysema (blue).

The second, which looked specifically at the genomes of people with COPD, was led by Phuwanat Sakornsakolpat at Brigham and Women's Hospital in Boston, Massachusetts. It compared data from about 36,000 people with COPD with data from some 222,000 healthy people. The researchers found 82 regions associated with COPD, some of which had not previously been linked with the disease. Of these, 22 regions have never been associated with lung function. The identification of these additional genetic loci in people with COPD suggests that there might be factors that affect the development of the disease, but that do not measurably affect lung function.

These studies underline the genetic diversity of COPD and reveal more of the genetic factors that make some people more susceptible to the disease.

Nature Genet. 51, 481-493 (2019);

Nature Genet. 51, 494-505 (2019)

\section{Imagingbiomarker validated in humans}

A non-invasive imaging technique that can characterize different types of COPD pathology has been shown to be accurate when compared with results of tissue-sample analysis. A team has shown that the technique, based on computed tomography (CT) scanning, can differentiate disease affecting the small airways from emphysema (damage to air sacs called alveoli). Evidence suggests that damage to small airways precedes emphysema, and might accumulate for years before it is detected through a lungfunction test. The new technique could identify people with early stage disease, when treatment might be the most effective.

CT scans can already detect enlarged alveoli that cause emphysema by measuring lungtissue density when the lungs are full. Small-airway disease also alters tissue density by trapping gas, but conventional CT does not have sufficient resolution to visualize these airways, which are about 2 millimetres in diameter.

The new method, called parametric response mapping (PRM), compares CT scans conducted after inspiration and expiration to calculate changes in lung-tissue density that indicate small-airway disease. The team says PRM allows it to differentiate between density changes associated with emphysema and those that signal damage to the small airways.

Meilan Han at the University of Michigan, Ann Arbor, led the collaboration of radiologists and surgeons to test the accuracy of the technique on tissue removed from the lungs. The team conducted PRM in 11 people with severe COPD before lung transplant surgery. The researchers then used micro-CT - an ultra-high-resolution technology that cannot be used on people owing to high energy X-rays - to analyse lung tissue 
samples. Regions designated as small-airway disease by PRM corresponded to characteristics of that condition - the loss, narrowing, thickening and obstruction of the bronchioles.

Future studies will need to show that PRM is accurate in milder disease. But it could be used to identify smokers at risk of developing COPD, or track the impact of new therapeutics on disease progression.

Am. J. Respir. Crit. Care Med. 200, 575-581 (2019)

\section{Phase III trial for nerve therapy begins}

A therapy that disrupts nerves in the lungs has entered clinical trials to test its efficacy in preventing flare-ups of COPD.

Many people with COPD have periods of symptom exacerbation, which can lead to hospitalization. Targeted lung denervation (TLD) aims to reduce flare-ups by delivering a radiofrequency electrical current that disrupts nerves in the lung. The technique is based on research suggesting that the neurotransmitter acetylcholine causes airway constriction, excess mucus and inflammation in COPD. TLD is intended to produce a longer-lasting effect than anticholinergic inhalers, which deliver a chemical to block the action of acetylcholine, by disrupting the production of acetylcholine by nerves.

In a double-blind, placebocontrolled phase II trial led by Dirk-Jan Slebos of the University of Groningen, the Netherlands, the technique was shown to be safe. Half of the 82 participants with COPD received TLD therapy alongside their existing treatments. Between 3 and 6.5 months after treatment, $32 \%$ of those treated had respiratory adverse events, compared with $71 \%$ of people in the placebo group. Over a year, the risk of hospitalization was also much lower in the treatment group.
Outcomes, such as patientreported symptoms, were not significantly different - but a phase III trial, aiming to enrol 520 people, is now under way and might prove more sensitive.

Am. J. Respir. Crit. Care Med. 200, 1477-1486 (2019)

\section{Blood-cell secretions sparksymptoms}

Small packages of molecules known as exosomes have been found to cause the damage to lung tissue seen in COPD. A study led by Edwin Blalock at the University of Alabama at Birmingham showed that exosomes released by white blood cells called neutrophils generate COPD pathology in the lungs of healthy mice.

Exosomes are secreted by all cells, and are involved in cell signalling or transporting cargo such as enzymes. Neutrophils become activated when they detect an infection. When this happens, they shed exosomes covered in the protein-degrading enzyme neutrophil elastase. In the lungs, this breaks down collagen and elastin in the extracellular matrix - the scaffold that supports the grapelike structures known as alveoli.

Ordinarily, the lungs are protected against this damage by $\alpha 1$-antitrypsin, which inhibits neutrophil elastase. However, the team found that the enzyme escapes this inhibition when bound to an exosome. The exosomes also carry another surface protein called Mac-1 that binds to collagen fibrils, increasing the exosome's capacity to damage tissue. As a result, exosomes from activated neutrophils were found to be 10,000 times more damaging than neutrophil elastase alone.

The team showed that when activated neutrophil exosomes were collected from the lung fluid of people with COPD and transferred to mice, they enlarged alveoli and increased airway resistance. Dislodging neutrophil elastase from exosomes, or inhibiting the enzyme or Mac-1, could be potential treatments.

Cell 176, P113-P126 (2019)

\section{Bioengineered lung transplants advance}

Lung transplantation is a

last-resort therapy for end-stage COPD. But the supply of donor organs is limited, and transplants can be rejected. Bioengineered lungs might offer a solution. A team led by Joaquin Cortiella at the University of Texas Medical Branch at Galveston has reported the most advanced attempt so far to transplant bioengineered lungs into pigs.

Previously, transplanting bioengineered lungs into rats resulted in haemorrhage, coagulation and swelling, so the team's main aim was to produce a working structure of blood vessels that could support longterm survival of transplanted tissue. To create the lungs, the team stripped pig lungs of cells, leaving the extracellular matrix, before repopulating these 'scaffolds' with cells derived from recipients' lungs. It then added plasma and proteins to develop the lungs in culture over 30 days.

Once transplanted into pigs, the lungs developed vascular tissue and blood circulation, as well as alveolar tissue. There were no issues with rejection, and the pigs survived for up to two months. The organs had similar gene expression and immune function to the pigs' own lungs, and native microorganism communities established themselves in the organs.

The lungs did not contribute to gas exchange, because the bioengineered vasculature was not linked to host arteries. Nevertheless, this is the longest an animal has survived in a study of this kind. Extracellular lung components are highly conserved between pigs and humans, so it is possible that pig scaffolds could be used in human transplants.

Sci. Transl. Med. 10, eaao3926 (2018)

\section{Corticosteroids disrupt microbiome}

Inhaled corticosteroids might allow bacterial infections to flourish by altering the community of microorganisms in the lung. A team led by Sebastian Johnston at the National Heart and Lung Institute, Imperial College London, showed that steroids disrupt lung microbiota by inhibiting antibacterial molecules, which might explain previous studies that indicate the drugs increase the risk of pneumonia in people with COPD. The team analysed sputum samples from people with COPD to show that inhaled steroids are associated with disrupted lung microbiota and proliferation of Streptococcus bacteria. When mice were given steroids, there was a similar increase in bacteria.

In human cells and mouse models, the team showed that steroids impair the clearance of Streptococcus pneumoniae, the most common bacterial cause of pneumonia, by suppressing the antimicrobial peptide cathelicidin. The steroids seem to increase expression of a protease that degrades cathelicidin, called cathepsin D. The team also showed that cathelicidin reversed the increased bacterial load seen in steroid-treated mice, as did inhibiting cathepsin $\mathrm{D}$. These strategies could be effective in preventing or treating COPD complications.

Science Trans. Med. 11, eaav3879 (2019)

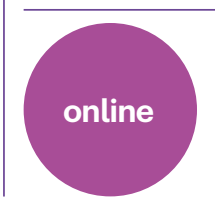
com/2xyawlx 